Instituto Internacional de Investigación y Desarrollo Tecnológico Educativo INDTEC, C.A.

DOI: https://doi.org/10.29394/scientific.issn.2542-2987.2017.0.0.17.317-335

OAI-PMH: http://www.indteca.com/ojs/index.php/Revista Scientific/oai

\title{
Responsabilidad Social en el Desempeño Laboral del Docente en el Contexto de la Educación Básica del Nivel Medio General
}

\author{
Autoras: Olga Josefina Álvarez Enríquez \\ Universidad Nacional Experimental "Rafael María Baralt", UNERMB \\ olgajosefina.62@gmail.com \\ Zulia, Venezuela \\ María Elena Rojas Bravo \\ Universidad Nacional Experimental "Rafael María Baralt”, UNERMB \\ marielenaro@gmail.com \\ Zulia, Venezuela
}

\section{Resumen}

La presente investigación tuvo como propósito general, analizar la responsabilidad social presente en el desempeño laboral del personal docente a nivel de educación media general en la U.E. Virgen del Monte Carmelo del Municipio Valmore Rodríguez. Se insertó el método cualitativo etnográfico. Para la recolección de información, se aplicó la técnica de observación y la entrevista a profundidad, las cuales fueron procesadas a través de la categorización, la estructuración individual y general, la contrastación y la teorización. La validez y la confiabilidad permitieron confirmar los hallazgos encontrados, evidenciando la necesidad de la preparación permanente del docente en cuanto a la atención y formación del estudiante en correspondencia con lo planteado en el currículo a nivel de media general, así como la sensibilización hacia el desarrollo de una práctica pedagógica. Generando una aproximación teórica acerca de los ejes temáticos relacionados con la responsabilidad social del docente, las características, los conocimientos e Interpretación de los signos y significados que le atribuyen los actores sociales a la responsabilidad social en su desempeño laborar. Los resultados del estudio condujeron a una serie de reflexiones tan importante al develar que existe una praxis pedagógica cerrada, e impuesta por los docentes, sin tomar en cuenta la formación del estudiante, destacándose en una sencilla y simple trasmisión de conocimientos desde la perspectiva de la responsabilidad social.

Palabras clave: responsabilidad social; desempeño laboral; compromiso. 


\title{
Social Responsibility in the Work Performance of the Teacher in the Context of Basic Education of the General Middle Level
}

\begin{abstract}
The current research had like general to purpose to analyze the social responsibility presents in the teacher's job performance in the general half education level, in the school educational Unit Monte Carmelo Virgin, of the Valmore Rodriquez District. Is insected the ethnographic cualitative method. To gather information, was aplicated the observation technique and the interview in depth, which were processed through the categorization, the genral and individual structuring, contrasting, and theorizing. The validity and confiability let to confirm findings showing the necessity of the teacher's permanent preparation in attention and training of the student in relationship to the expounded in the curriculum of general half level, so as the sensitization to the development of a pedagogical practice. Generating a theoric approximation of the mains themes relationed with the social responsibility of the teacher, the characteristies, the knowledges and interpretation of signes and meanings attributed by the social actors to the social responsibility in their job performance. The results of the study drove to a series of reflextions so important to show to exists a close pedagogical practice and imposed by the teacher's, no taking the training of the student, emphasing a casy and simple knowledges transmission from the social responsibility perspective.
\end{abstract}

Keywords: social responsibility; job performance; commitment. 


\section{Introducción}

Comprender el proceso educativo que se desarrolla en los actuales momentos, considerando también la estructura social y analizando los aspectos como las condiciones sociales, demanda la formación de un ser cónsono orientado hacia la consolidación de una sociedad humanista, capaz de hacer frente a las oportunidades que se presentan para hacer de ella una herramienta útil, con miras a una mejor calidad de vida.

Ahora bien, nuestro país hoy día vive un estado de profundo cuestionamiento sobre su convivencia y bienestar, es un momento serio y radical sobre el futuro como el presente de miles de ciudadanos que se forman en nuestras escuelas con la esperanza de una vida mejor. Es por ello que se realiza esta investigación etnográfica referente a la responsabilidad social del docente en su desempeño laboral, con el propósito de conocer los objetivos fundamentales transversales formulados en los planes educativos, el cual buscan ser una respuesta a las necesidades de una educación que necesita formar ciudadanos comprometidos con el desarrollo de su país y el bienestar de todos quienes habitan en él.

De esta manera, muchas de estas responsabilidades recae firmemente en los miembros que participan y hacen vida en las instituciones educativas; pues la importancia del rol del docente en el quehacer diario marca en su accionar una diferencia en el provenir de los estudiantes, cuando incluyen en los contenidos programáticos temas comprensivos humanistas, al intercambiar experiencias, diálogos con los saberes propios de la comunidad, ofreciendo la oportunidad de detenerse a observar, conocer el contexto cultural y personal de los estudiantes, cuyo propósito de los proyectos académicos sea diseñar formas de trabajos en conjuntos para mejorar las condiciones de la familia por ende la comunidad.

Es por ello que, el docente tiene la gran responsabilidad de atender las necesidades que demanda la sociedad en la cual se encuentra inmersa la 
institución educativa, preparado de forma integral, con valores y principios para aportar elementos positivos, con miras hacia la calidad educativa, consciente de sus funciones, donde su responsabilidad se vea orientada al servicio hacia los demás, para comprender e interpretar los intereses y las necesidades de los estudiantes.

Por lo tanto, es imprescindible que el líder educativo se fortalezca mostrando una profunda apreciación por la libertad humana, buscando un cambio en la forma de actuar, precedido de una transformación de cómo se piensa y se siente, ya que la responsabilidad que se requiere en estos tiempos demanda un cambio de paradigma en las instituciones educativas.

En este sentido, la responsabilidad social no debería estar encaminada como una alternativa para adquirir los recursos requeridos y subsistir en la vida; sino que además, como una fuente de satisfacción mutua, que induzca a los estudiantes al anhelo de aprender para que proyecten la confianza hacia los demás, que se introduzca en ese mundo extraño pero real del niño, que ayude a comprender y ordenar sus ideas, con un seguro y sentir familiar, que encamine la efectividad educativa del plantel donde hacen vida participativa.

En consecuencia, la realidad educativa, exige una actitud y un carácter histórico de crear y reinventar, como acción formativa desde una visión humana a través de la responsabilidad social en el rol asumido por el docente, que le permita auto reflexionar en su proceder y penetrar a un mundo más complejo en el cual se vive, a fin de develar las incompatibilidades existentes entre los seres humanos.

\section{Presentación de la Realidad Estudiada.}

Por consiguiente, la investigación se realizó en la U.E. "Virgen del Monte Carmelo" la cual es dependencia del Ministerio del Poder Popular para la Educación, ubicada en la avenida 7 de Bachaquero del Municipio Valmore Rodríguez del Estado Zulia - Venezuela. Fue fundada en el año 1998, por la 
directora Amalia Vivas con ayuda de su esposo (Juan Moreno),

Actualmente, la institución cuenta con una matrícula de 1060 estudiantes, conformando los tres niveles de educación básica: Educación Inicial con 130 estudiantes; Educación Primaria 550 estudiantes, y Educación Media General 380 estudiantes. De igual manera forman parte como equipo del personal, 57 docentes, 03 directivos, 04 administrativos, 12 obreros, y 15 cocineras escolares.

Dentro de este marco de ideas, esta fundación ocurre con la participación de los padres y representantes e incorporación paulatina de los organismos públicos y privados de la localidad, mostrándola como ejemplo a seguir por las otras instituciones de la jurisdicción, por los resultados y las respuestas efectiva a las exigencias planteadas por el sistema educativo venezolano con el firme propósito comunitario de poder contar con la planta física completamente independiente.

\section{Aporte de la Investigadora}

El sistema educativo, es el medio esencial e integral de la formación de toda persona para poder ser incorporado a la vida así como la base fundamental en la transformación de un país; puesto que socialmente los requerimientos en el campo de trabajo son cada día más marcados, lo que demanda de expertos competentes que den respuesta a los problemas de una realidad compleja y dinámica, donde la incertidumbre forma parte de la cotidianidad, demandando de ellos que adopten en su trabajo, una actitud reflexiva, crítica y de gestión con respecto a la realidad educativa, pero que además posean capacidades de investigar científicamente la forma de cómo transformarla creativamente.

Es por ello, que se requiere de un personal educativo comprometido, responsable y humanista, que vaya más allá de las necesidades del estudiante, con consciencia clara de su función de formar futuros individuos 
para la vida, a quien le compete cumplir con las exigencias emanadas por el Estado Venezolano.

Para ello se necesitan personas que asuman el reto como pensadores, tal como sostiene Freire, P. (1996), "realicen la tarea permanentemente de estructurar la realidad, de preguntarle y preguntarse sobre lo cotidiano y evidente, tarea ineludible para todo trabajador social”. (pág. 123). Lo que de alguna manera evidencia que los especialistas dedicados a la formación de los futuros ciudadanos aptos para la vida en su integralidad, al interactuar con su entorno y con los demás.

Ante esta realidad se puede detectar que, U.E. "Virgen del Monte Carmelo" presentan ciertas debilidades en cuanto la responsabilidad social del desempeño laboral del docente con respeto a la toma de consciente de formar futuros ciudadanos capaces de enfrentar los retos de la vida, garantizado la formación de nuevas generaciones de individuos interesados en lograr un estadio de conocimiento del mundo y en particular al mejoramiento de sus entornos; reconociendo la docencia una práctica con sentido humano orientado a potenciar la razón tantos en unos, como en otros, el cual radica en la voluntad de los individuos por compartir un conocimiento.

\subsection{Inquietud que Orienta la Investigación}

El abordaje del presente trabajo de investigación surge a través de la inquietud que se generó por diferentes situaciones visualizadas con preocupación antes los acontecimientos ocurridos en los últimos tiempos en las organizaciones educativas, con respeto a la formación académica de los estudiantes del liceo; quien son estimados como seres íntegros, constructores de su propio conocimiento, a quien no se le puede negar la posibilidad de interactuar con su entorno, siendo capaz de poner en práctica la capacidad de analizar y reflexionar sobre lo que acontece a su alrededor a través del derecho que la concede la educación. 
Por tal motivo la situación presentada, conducen a plantear previamente las siguientes preguntas antes lo que reflejan algunos escenarios de los liceos públicos nacionales en el Municipio Valmore Rodríguez ¿Qué está ocurriendo con los estudiantes de educación media general, que frecuentemente están fuera del aula de clases y del plantel en horas escolarizadas? ¿Será que su horario de clases les permite contar con esas horas libres? ¿Están siendo debidamente atendidos estos jóvenes adolescentes por sus profesores asignados? ¿Están recibiendo los estudiantes su formación académica que como derecho le corresponde y que está contemplado en la Constitución de la República Bolivariana de Venezuela y en la ley Orgánica de Educación? ¿Cuál es el significado social del docente-educador o de un formador de nuevos humanos?

De este modo, al llevar a cabo el acercamiento interactivo como observador participante, con el propósito de conocer más de cerca el contexto real, se realizó la visita a la U.E. "Virgen del Monte Carmelo" el día 17-11-2015, donde primeramente se conversó formalmente y se solicitó el permiso a la directora Lcda. Amalia Vivas para realizar el proceso de investigación, así mismo dio a conocer las características generales del plantel lugar de estudio, como la historia de la institución, su gestión, visión y misión.

Ahora bien, es a través de estas interacciones y como sujeto cognoscente que aprende de esta realidad; qué en el mes de febrero se realizaron reuniones de consejos docentes de media general donde varias veces se dio la oportunidad de conocer y escuchar narrativas informales de los actores refiriéndose a los alumnos con expresiones tales como: "los estudiantes no tienen interés por estudiar y si sus padres no se dan mala vida por sus hijos yo no me la voy a dar", "yo no voy a descuidar a mis hijos, sólo por venir a cuidar una pila de delincuentes, que lo que vienen es a echar bromas y a colmarle la paciencia a uno", "con la situación que hay, no me voy a dar más mala vida". 
Evidentemente que estas actitudes contradicen el deber ser del docente, quien es el eslabón principal en el proceso de formación del hombre, su función como orientador y transformador de sociedades le delega la responsabilidad de la acción pedagógica; puesto que su rol primordial es ser facilitador y guiar al estudiante a construir y reconstruir sus propios conocimientos sobre la base de suscitar experiencias conforme con el nivel de desarrollo de los mismos a fin de contribuir con la educación integral del futuro ciudadano.

\section{Pertinencia Teórica}

En este sentido el modelo educativo nacional tiene como único fin garantizar una educación participativa, protagónica, democrática, libre; generadora de un modelo de desarrollo endógeno sustentable, que resalte las potencialidades del ser humano, bajo los principios de libertad, igualdad, justicia social; igualmente preparar la escuela en un espacio para la paz, donde el ejemplo de todos se fundamente en los valores del respeto, la tolerancia, solidaridad y el derecho humano.

De igual manera, la responsabilidad social en la educación tiene su impacto producto de la refundación de la República Bolivariana de Venezuela, sustentada en la Constitución, donde se plantea construir una sociedad con equidad e igualdad de oportunidades, así como la formación de un ciudadano comprometido con el futuro de su país. La educación se concibe entonces como un derecho humano y un deber social de toda persona, cuyo proceso es corresponsabilidad del estado, la familia y la sociedad.

Por lo que la educación secundaria bolivariana, tiene como finalidad lograr la formación integral de los y las adolescentes y jóvenes, atendiendo a los fines y principios que inspiran a integrarles la República Bolivariana de Venezuela; dando continuidad a los estudios primarios de éstos y 
permitiéndoles su incorporación al proceso productivo social, al mismo tiempo que los orienta para la prosecución de estudios superiores.

La finalidad de los estudiantes que egresan de la educación media general, es lograr que cada uno de ellos salga preparado, con una conciencia histórica e identidad venezolana, que alcance el desarrollo de su capacidad y habilidades para un pensamiento crítico, cooperativo, reflexivo y liberador que le permitan, a través de la investigación, contribuir a la resolución de problemas de la comunidad local, regional y nacional de manera corresponsable, solidaria, de libertad, con sentimiento de paz y consciencia humana.

Por otra parte, Martínez, M. (2008) manifiesta que "en la escuela los seres humanos pasan por un proceso de formación, en el que se posesiona de una sucesión de conocimientos pero se repara poco a nuestras características individuales y la particularidad del pensamiento, y donde el alumno tiene que hacer lo que el docente le dice $y$, en este sentido el buen alumno es aquel que puede ir formándose y aprendiendo de las asignaturas funcionales que el plantel entrega, pero que el docente no es capaz de aceptar fácilmente las particularidades de los estudiantes". (pág. 56)

Por otro lado, Abraham M. (2008) refiere que, "la responsabilidad social focaliza la atención de los directivos como en los miembros de una institución hacia los impactos (a efectos directos o colaterales) de las toman de decisiones en las actividades que pueden ocasionar hacia dentro y fuera de la organización. Se trata antes que nada de diagnosticar, cuidar y prevenir los impactos negativos posibles, de manera de maximizar los positivos para que la organización pueda ser considerada como socialmente pertinente (útil y beneficiosa para la sociedad en todas sus operaciones)" (pág. 304).

El mismo autor, manifiesta que la responsabilidad social se relaciona con la mejor predisposición y pro-actividad frente a los intereses de las diferentes personas o grupos con lo que una institución se vincula, mediante 
una adecuada gestión de los impactos que generan sus decisiones y actividades, respecto de los intereses y necesidades de las diferentes partes interesadas directa o indirectamente con su misión, desde perspectivas tan diversas como la economía, lo social, el medio ambiente o lo cultural.

Esto explica que los miembros de una institución educativa deben tener la capacidad de difundir y poner en práctica un conjunto de principios y valores que implica tener que canalizar múltiples intereses de la institución que conlleven a lo posible transformación social.

\subsection{Impacto de la Responsabilidad Social en Educación Media General}

La responsabilidad social en la educación tiene su impacto producto de la refundación de la República Bolivariana de Venezuela, sustentada en la Constitución, donde se plantea construir una sociedad con equidad e igualdad de oportunidades, así como la formación de un ciudadano comprometido con el futuro de su país. La educación se concibe entonces como un derecho humano $y$ un deber social de toda persona, cuyo proceso es corresponsabilidad del estado, la familia y la sociedad.

El artículo 102 de la Constitución Bolivariana de Venezuela establece que la finalidad de la educación:

Es un derecho humano y un deber social fundamental, es democrática, gratuita y obligatoria. El estado la asumirá como función indeclinable y de máximo interés en todos sus niveles y modalidades, y como instrumento del conocimiento científico, humanístico y tecnológico al servicio de la sociedad (pág. 3536).

En este sentido el modelo educativo nacional tiene como único fin garantizar una educación participativa, protagónica, democrática, libre; generadora de un modelo de desarrollo endógeno sustentable, que resalte las potencialidades del ser humano, bajo los principios de libertad, igualdad, justicia social; igualmente preparar la escuela en un espacio para la paz, donde 
el ejemplo de todos se fundamente en los valores del respeto, la tolerancia, solidaridad y el derecho humano.

La finalidad de los estudiantes que egresan de la educación media general, es lograr que cada uno de ellos salga preparado, con una conciencia histórica e identidad venezolana, que alcance el desarrollo de su capacidad y habilidades para un pensamiento crítico, cooperativo, reflexivo y liberador que le permitan, a través de la investigación, contribuir a la resolución de problemas de la comunidad local, regional y nacional de manera corresponsable y solidaria.

En este sentido el perfil de la educación bolivariana, tiene como centro al hombre como ser social y capaz de responder y participar activamente en la transformación de la sociedad en la que vive.

Es de acotar que la educación supone un proceso de aprendizaje y desaprendizaje, es decir, un proceso de desestructuración, puesto que ninguna realidad es inmutable ni perene, dicho de otro modo, los docentes son responsables de lo que suceda o no suceda en el campo educativo.

\subsection{Desempeño Laboral del Docente Socialmente Responsable}

La importancia de la función del docente se destaca en la medida que se tome conciencia al interpretar y hacer prevalecer el propósito de la educación, ya que representan el motor de la institución, para que los cambios esenciales de la mejora educativa se lleven a cabo.

Según Gibson, Ivancebich y Donellys (2007): sostienen que "el desempeño docente es el resultado o variable de la conducta, la estructura y los procesos organizacionales". (pág. 585) En lo planteado se entiende que toda institución orientada al servicio es sumamente importante, que todos los miembros pongan su mejor esfuerzo, teniendo una visión fija en el correcto, en adecuado, en el desempeño de sus acciones con la finalidad de lograr las metas y objetivos propuestos. 
Cochran y Lytle (2003), expresan:

El buen desempeño escolar no es otra cosa sino la capacidad que tienen las instituciones educativas de cumplir con los objetivos planteados según el ordenamiento jurídico venezolano, en los tiempos que corresponden, con métodos acordes con los principios y valores consagrados en la constitución. El desarrollo de los parámetros para examinar, reconocer y estimular una buena práctica educativa es una obligación del estado que nos permite ponernos de acuerdo en cuales deben ser los criterios estandarizados para calificar las instituciones educativas como buenos y sus exámenes periódicos facilitan el diagnostico permanente del sistema educativo para favorecer el diseño de políticas más adecuadas para mejorar la calidad (pág. 65).

En estos términos, la constitución de la república bolivariana de Venezuela establece que la educación y el trabajo son los medios fundamentales para lograr una sociedad justa, libre, solidaria, partiendo de la escuela como espacio para la paz, orientada a inculcar valores.

\section{Metodología de la Investigación}

Esta investigación se inscribe en el paradigma cualitativo donde no existe una realidad lineal de los fenómenos en estudio, donde el objetivo final del proceso investigativo es analizar el comportamiento de los docentes en ámbito laboral comprender su manera de pensar y lo que sienten; la finalidad es describir y explicar las razones de los diferentes aspectos del comportamiento, que motivan al hombre actuar en su ambiente. En sí el paradigma representa la manera de observar al mundo, de explicar y comprender la realidad, dentro del marco referencial de ellos mismos y de experimentar la realidad como los actores la experimentan, buscando una comprensión detallada de las perspectivas de las personas involucradas con la investigación. 
El paradigma que se utilizó en la presente investigación es el cualitativo el cual justifica porque con él se puede analizar, comprender e interpretar el mundo de relaciones que se plantean en el estudio como es el de generar un constructo teórico de ejes temáticos relacionados con la responsabilidad social del docente en el desempeño docente, de allí que se fundamentó en el método etnográfico, el cual permite comprender la complejidad estructural de los fenómenos que viven y sienten las personas involucrada en el ejes problemáticos asociados a su cotidianidad, involucrados como coinvestigadores de su propia realidad y de su propio medio.

Al respeto, Martínez (2009), señala que:

La etnografía es una rama de la antropología que estudia descriptivamente las culturas; etimológicamente el estilo de vida de un grupo habituadas a vivir juntas (ethnos). Por lo tanto, ethnos, sería la unidad de análisis para el investigador constituido por un grupo humano que constituye una entidad cuyas relaciones estén reguladas por la costumbre o por ciertos derechos y obligaciones recíprocos (pág. 181).

Tomando en cuenta que el objeto de estudio, abordado en este caso, la institución educativa "Virgen del monte Carmelo" como la unidad social que pude ser estudiada etnográficamente ya que se comparte o se guían por formas de vida y situación que los hacen semejantes.

Es de acotar que a través del método etnográfico permitió al investigador, mediante la observación describir las múltiples formas de vida del desempeño laboral del docente, lo que hacen, como se comportan y cómo interactúan entre sí, describir sus creencias, intereses, valores, motivaciones, perspectivas y como estos pueden variar en diferentes momentos y circunstancias.

De igual forma para la selección de los informantes clave en este trabajo de investigación cualitativa, se contó miembros clave y privilegiados en cuanto a su capacidad informativa, de apoyo más importantes, ya que a través de 
ellos se fundamentan los modelos, teorías y propuestas para el desarrollo de este estudio. De igual forma se utilizó la técnica para recolectar información de modo alternativo y complementario, más empleadas en esta dimensionalidad, epistémica, como un triángulo cualitativo referido al abordaje como: la observación participante, la entrevista en profundidad, y la historia de vida.

Asimismo, se hizo uso de varias técnicas y recursos como la cámara fotográfica para captar imágenes fijas de los encuentros y escenarios, además, se llevó un diario de entrevista para tomar notas pormenorizadas de las circunstancias, conductas no reveladas, como gestos, expresiones, posiciones corporales, entre otros, y para complementar, este proceso se utilizó como instrumento de registro la grabadora de audio.

A medida que se obtuvo los datos se generan supuestos, realizando múltiples análisis (reinterpretando). El investigador pone el énfasis en la construcción o generación inductiva de categorías que permitan clasificar los datos recogidos en base al análisis de la temática común, al analizar la responsabilidad social del docente en educación media general. Luego buscara desarrollar una compresión sistemática del contexto estudiado a partir de los términos y palabras de los propios miembros.

De esta manera el autor, presenta los siguientes procesos que implica: la categorización, la estructuración individual y general, la contrastación y la teorización propiamente, y por último el tratamiento de la información recibida sobre la base de la información aportada, a través de las entrevistas apreciadas y después de haber recolectado la información aportada por los diferentes informantes, se pudo determinar con el extenso proceso de categorización.

Por otra parte, el procedimiento de contrastación contribuyo, para lograr la confiabilidad y la validez del estudio entre los elementos teórico, puesto que los resultados obtenidos en el ámbito de estudio y la interpretación de ambos, revelaron que sus resultados no obedecen de la forma utilizada para recabar 
y analizar los hallazgos, sin embargo, fue de mucha utilidad y enriquecedor las reflexiones finales, concediendo mayor validez en la exactitud de la investigación.

\section{Reflexiones Finales}

Este contexto abordado de la investigación está orientado a concretar los hallazgos pertinentes a la intencionalidad de la cual se inició para su desenvolvimiento del discurso de los informantes claves manifestando en las entrevistas, para confirma un conocimiento del tópico responsabilidad social en el desempeño laboral del docente, en el cual se constituye el adecuado sentido y significados que especifican las vivencias familiares, sociales, educativas y profesionales actuales en la vida diaria.

En el análisis e interpretación de los resultados obtenidos por los informantes claves consintieron en llegar al método de la teorización, siento la intención de este período investigativo, de manera que la responsabilidad social emprendido por la investigadora, se desarrolle de manera más amplio desde el ámbito tanto ontológico, epistemológico y axiológico indiferentemente desde el paradigma que se tome para enfrentar, bien sea el cualitativo 0 cuantitativo. Enfocándose en el paradigma cualitativo va a depender de la complejidad del hombre ya que su estudio es inconcluso, considerando la diversidad de emociones presentadas a través de las circunstancias del diario vivir.

Refiriéndose en este caso Morín (2003), que "el desafío de la complejidad es de pensar complejamente como metodología de acción cotidiana, cualquiera que sea el campo donde desempeña su accionar". (pág. 127). En función a lo acordado se constituye en un conjunto de concepciones ante la forma real de estudiar el comportamiento del hombre desde el lugar donde labora su quehacer diario. 
Considerando, que el hombre por su naturaleza es un ser complejo, variante en su acontecer diario, por lo tanto no se debería estudiar de forma independiente de su ambiente o de todo lo que le rodea, es decir, tomarlo en cuenta sin tener contactos con otros es desconocer su condición, puesto que la ciencia que estudia antropología así lo establece, y plantea que la esencia humana a la verdad de esta ciencia involucra escudriñar todo lo que atañe al hombre en todo su ambiente personal y social, es decir todos los recaudos, datos que acceden a revelar las categorías y las sub-categorías para así correlacionar entre ellas con el propósito de conseguir la información de lo interpretado, desde una dimensión ontológica desde sus diferentes aspectos y de acuerdo a la intención del estudio de la investigadora.

Por lo tanto, agrupando las informaciones recolectadas obtenida del trabajo de la relación entre el personal docente entrevistado de la U.E. "Virgen del Monte Carmelo", desde el momento que se dan los significados referentes al conocimiento de responsabilidad social del desempeño laboral del docente, a partir del cual se acota que la interrelación humana, es debido a la convivencia que se aprende en función a los valores, representando la más alta esperanza de la estabilidad del ser humano.

Siendo incuestionablemente una condición de vida incorporada al entrenamiento y a la admiración de valores humanos de amor, de verdad, libertada, viviendo cada día compartiendo y transfiriendo con su espíritu afecto, dulzura, paciencia y compromiso ético, de responsabilidad; en especial en el contexto que rige la responsabilidad social ética, enfocada en la moral y en la necesidad que presiden a la responsabilidad del individuo en la actual sociedad.

\section{Referencias}

Abraham, M. (2008). El servicio comunitario y la responsabilidad social universitaria. Una oportunidad para reflexionar desde y con la 
universidad. Editorial universidad de Carabobo. 1era edición. Valencia, Venezuela.

Cochran-Smith, y Lytle, S. (2003). Más allá de la incertidumbre escolar: adoptar una actitud indagadora sobre la práctica. Barcelona: Octaedro.

Constitución de la República Bolivariana de Venezuela (2000). Gaceta Oficial Extraordinaria. No. 5.453. Venezuela.

Ministerio del Poder Popular para la Educación. (2007). Diseño Curricular del Sistema Educativo Bolivariano. CENAMEC. Caracas, Venezuela. Freire, P. (1996). Pedagogía de la Esperanza. 2da. Ed. México: Siglo XXI. Gibson, J. Ivancevich, J. y Donnelly J. (2007). Las Organizaciones. Décima Edición. McGraw-Hill. Santiago de Chile.

Martínez, M. (2009). Epistemología y Metodología Cualitativa en las Ciencias Sociales. México: Editorial Trillas.

Martínez, M. (2008). Aprendizaje servicio y responsabilidad social de las Universidades. Barcelona: MEC y Octaedro.

Morín, E. (2003). Introducción al Pensamiento Complejo. Editorial Gedisa. Barcelona, España. 


\section{Olga Josefina Álvarez Enríquez}

e-mail: olgajosefina.62@gmail.com

Nacida en Mene Grande. Estado Zulia, Venezuela.

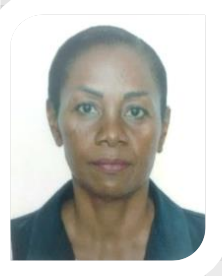

Cursa estudio de Doctorado en Ciencias de la Educación

en la Universidad Nacional Experimental Rafael María

Baralt, Estado Zulia. Magister Scientiarum en

Administración de la educación Básica en la Universidad

Nacional Experimental Rafael María Baralt, Estado

Trujillo (2006). Licenciada en Educación Mención Integral en la Universidad Nacional Experimental Rafael María Baralt (2000). Actualmente se desempeña como Subdirectora Administrativa en la U.E. "Virgen del Monte Carmelo", en el Municipio Valmore Rodríguez Estado Zulia, desde el 2000 hasta la actualidad. Asistió a jornadas de formación en congresos pedagógicos como participante a nivel nacional, regional y municipal. 


\section{María Elena Rojas Bravo \\ e-mail: marielenaro@gmail.com}

Nacida en Coro. Estado Falcón, Venezuela. Cursa estudio de Doctorado en Ciencias de la Educación en la Universidad Nacional Experimental Rafael María Baralt, Estado Zulia. Magister Scientiarum en Administración de la educación Básica en la Universidad Nacional Experimental Rafael María Baralt, Estado Trujillo (2006). Licenciada en Educación Mención Integral en la Universidad Nacional Experimental Rafael María Baralt (2000). Actualmente se desempeña como Subdirectora Académica en la U.E. "Virgen del Monte Carmelo", en el Municipio Valmore Rodríguez Estado Zulia, desde el 2001 hasta la actualidad. Se desempeñó durante tres años como docente en Educación Media general U.E.N Néstor Luis Negrón, Municipio Valmore Rodríguez del Estado Zulia. Experiencia como docente en el Instituto Universitario de Educación Especializada (IUNE). Asistió a jornadas de formación en congresos pedagógicos como participante y como ponente a nivel nacional, regional y municipal. Asimismo, se desempeña como coordinadora de formación permanente en la U.E. "Virgen del Monte Carmelo", Estado Zulia. 